\title{
Crossing the germline: CRISPR-Cas9 and our responsibility as reproductive endocrinology and infertility physicians
}

\author{
Daniel J. Kaser ${ }^{1,2}$ • Jason M. Franasiak ${ }^{1,2}$
}

Received: 15 August 2017 / Accepted: 17 October 2017 / Published online: 30 October 2017

(C) Springer Science+Business Media, LLC 2017

\section{Introduction}

"Will it be possible to obtain son or daughter, according to specifications...?"

- Anonymous, "Conception in a Watch Glass", 1937 [1]

Eighty years after this question was raised in an anonymously penned editorial in the New England Journal of Medicine, largely attributed to the giant Dr. John Rock, we may have an answer. The clustered regularly interspaced short palindromic repeat (CRISPR)-associated system (Cas) geneediting technology now has been applied to gametes and triploid and diploid human embryos. The ease, speed, and low cost of the system have been hailed by many scientists as a tremendous breakthrough. The possibility that this technology, in the not too distant future, could be applied clinically for primary prevention of disease indeed may appeal to doctors and patients alike. Serious concerns remain, however, regarding both the ethics of the technology and technical limitations including its efficiency and fidelity [2-4].

Authors from a prominent gene-editing laboratory stated in a recent manuscript, "Separately from the debate of the merit and ethics of germline editing in humans, the feasibility of correcting genetic defects via CRISPR technology in human zygotes has not been really tested" [4]. This view implies that

Daniel J. Kaser

dkaser@rmanj.com

1 Reproductive Medicine Associates of New Jersey, 140 Allen Road, Basking Ridge, NJ 07920, USA

2 Sidney Kimmel Medical College, Thomas Jefferson University, Philadelphia, PA 19107, USA ethics and technical advancement occur independent of one another; it seems vital, however, that the ethics of any disruptive technology be considered together with, if not before, the technical development itself. Unfortunately, history has shown that often technology is developed first, and then society reacts by accepting or rejecting it. Now, more than ever, our field is pressed with questions of where we are headed; should we, as infertility specialists, continue this line of investigation, and if so, under what circumstances is application of this technology clinically, morally, and socially acceptable? If we do not take the lead on this, which specialty will and to what end?

A 2017 joint report by the National Academies of Sciences, Engineering, and Medicine entitled "Human Genome Editing: Science, Ethics and Governance" seeks to address these questions and others [5]. This 261-page PDF is available for free download online (https://www.nap.edu/catalog/24623/ human-genome-editing-science-ethics-and-governance). There are 22 co-authors on this report, including medical geneticists, stem cell and cancer biologists, pediatricians, internists, lawyers, and bioethicists. Surprisingly, not a single reproductive endocrinology and infertility (REI) physician, arguably the one most likely to counsel patients about this technology if it were ever applied clinically, was involved in drafting this document.

\section{Moral quandaries and a call to action}

In the National Academies' report, the expert panel concluded that trials of germline gene editing should be allowed only if they meet certain criteria (Box 1), the first being "the absence of reasonable alternatives". How, then, does contemporary IVF with preimplantation genetic diagnosis (PGD) for single gene disorders not provide such a reasonable alternative for 
Box 1 Guidelines for clinical trials involving human germline editing as proposed by the National Academies of Science, Engineering, and Medicine

- Absence of reasonable alternatives

- Restriction to preventing a serious disease

- Restriction to editing genes that have been convincingly demonstrated to cause or to strongly predispose to the disease or condition

- Restriction to converting genes to versions that are prevalent in the population and are known to be associated with ordinary health with little or no evidence of adverse effects

- Availability of creditable pre-clinical and/or clinical data on risks and potential health benefits of the procedures

- Ongoing, rigorous oversight during clinical trials of the effects of the procedure on the health and safety of the research participants

- Comprehensive plans for long-term, multigenerational follow-up while still respecting personal autonomy

- Maximum transparency consistent with patient privacy

- Continued reassessment of both health and societal benefits and risks, with broad ongoing participation and input by the public

- Reliable oversight mechanisms to prevent extension to uses other than preventing a serious disease or condition

nearly all possible lines of investigation? Opponents of gene editing in human embryos might interpret this clause as a nonstarter for all efforts at clinical adoption. Others might disagree and argue that CRISPR-Cas9 may improve the yield of embryos suitable for transfer in PGD cases. Still, questions arise: What is the difference between a disease and a condition? Which diseases are considered serious, and who decides this? What infrastructure is needed for proper oversight to ensure gene corrections are limited to pathologic variants? What is the reference population for determining prevalence of a "normal" genotype? How will long-term follow-up be conducted effectively?

The authors of the National Academies' report miss the mark on other occasions, too; they speculate that data from CRISPR-Cas9 experiments of human embryos "is expected to inform and improve IVF procedures and embryo implantation rates and reduce rates of miscarriage" [5]. To be clear, CRISPR-Cas9 will neither improve implantation nor decrease rates of spontaneous abortion unless it is targeted to genes involved in such outcomes - its sole purpose at present would be to modify heritable causes of monogenic disease. It is conceivable that one day, single-gene mutations may be identified that are involved in embryogenesis or recurrent implantation failure or miscarriage and accordingly could be targeted with CRISPR-Cas9; in our opinion, though, currently there are no such candidate genes that would justify application of the technology for this purpose. Likewise, knowledge gained from experiments with CRISPR-Cas9 about mutagenesis and DNA repair may be applicable to other areas of medicine in the future, such as cancer or aging. Certainly, though, many would agree that the use of human embryos for the express purpose of serving as a scientific model would not be ethically permissible in cases where other appropriate models exist [6]. Thus, we are faced with the decision whether we should continue this research in order to prevent transmission of genetic disease to offspring. This momentous duty underscores the importance for leaders in our field to speak out, in favor or not, of this rapidly developing technology. If we decide to move forward with this clinically, let us do so transparently and not under the guise of improving implantation rates.

\section{Scientific shortcomings: target efficiency and mosaicism}

Questions likewise abound about the technology itself. Importantly, how often does the desired mutation find its way into the target genome? The targeting efficiency of the CRISPR-Cas9 system appears superior to other gene-editing techniques such as zinc finger nucleases (ZFNs) and transcription activator-like effector nucleases (TALENs), although these techniques set a low bar to reach with efficiencies in human cells ranging from 1 to 50\% [7-9]. Assuredly, this would be expected to improve with further research, but such inefficiency can produce mosaic cell lines within the embryo, in which some of the cells have been restored to wild type and others remain unrepaired.

US researchers reportedly have overcome major targeting inefficiencies and the challenge of mosaicism in edited embryos, though. In August 2017, Ma et al. published an innocuously titled report in Nature called "Correction of a pathogenic gene mutation in human embryos" in which they describe repair of a heterozygous four base pair deletion in $M Y B P C 3$, one genetic cause of hypertrophic cardiomyopathy [10]. The authors repaired 58/58 (100\%) of human preimplantation embryos. The group achieved this remarkable efficiency by co-injecting CRISPR-Cas9 with the mutant sperm at the time of ICSI of metaphase II wild-type oocytes, instead of injecting at the typical S-phase zygotic stage. By introducing CRISPR-Cas9 prior to fertilization, the editing occurred when there was only a single mutant copy of the targeted gene present. Cas9-induced double-strand breaks (DSBs) were repaired predominantly via homology-directed repair (HDR) using the corresponding wild-type maternal gene as the template instead of the injected oligonucleotide template. This critical protocol change allowed correction of all embryos with near elimination of mosaicism. Indeed, there was only one mosaic, and it would have still been eligible for transfer; its blastomeres were uniformly wild-type, but some had been repaired using the maternal allele as the template and others using the injected oligonucleotide template.

Of note, M-phase injection also allowed most (42/58, $72.4 \%$ ) embryos to be restored to wild type, so they were indistinguishable from unaffected embryos [10]. The 
remaining 16/58 (27.6\%) embryos were in fact corrected, though through an error-prone non-homologous end-joining (NHEJ) mechanism, which can lead to insertions or deletions "indels" at the break site. As a result, these 16 embryos would not be suitable for transfer. Four of six embryonic stem cell lines created from repaired blastocysts were wild-type, and two contained indel mutations resulting from NHEJ. Thus, while these results are promising in terms of nearly eliminating mosaicism, further work is needed to understand if DNA repair mechanisms can be further biased toward HDR to minimize the creation of indels.

Another important limitation highlighted by Ma et al. is the scope of conditions that could be addressed with M-phase injection and HDR. As the authors rightfully acknowledge, the correction of homozygous mutations, in which a wildtype allele would not be present to serve as a template, may prove more difficult unless further work improves the efficiency of repair using the injected oligonucleotide template.

A recent criticism of the Ma et al. study cast further doubt about whether the authors presented adequate evidence demonstrating correction of the pathogenic allele, or rather simply failed to detect the mutant allele and inferred that their inability to detect it was consistent with correction [11]. As this critique indicates, the sequencing data should have been phased with linkage analysis to conclusively prove that the wild-type allele from one of the maternal homologs was indeed incorporated into the paternal homolog. Short of doing this, the critics reason there is no direct evidence of gene correction, and alternative explanations like allele dropout from a large Cas9-induced deletion affecting a primer binding site, or even parthenogenesis, that could account for the reported findings [11]. Clearly, given the profound implications of clinically applying this technology, further work is needed to verify indisputably that the targeted mutation has been corrected.

\section{Off-target effects}

It is not only relevant to consider the efficiency of target correction, but also its fidelity. CRISPR-Cas9 is specific but is able to tolerate a mismatch in several nucleotides and still generate DSBs. Thus, in sites with only a few base pair difference, off-target DNA cleavage can occur due to this "wobble". These off-target effects represent a potential source of mutation in regions not intentionally targeted by the guide RNA that confers specificity to the Cas9 endonuclease.

When studying off-target effects in human embryos, whole genome sequencing (WGS) is required to fully quantify the error burden. However, some investigators only look at sites in the genome that are highly homologous to the intended site of repair as off-target effects may be enriched here. Importantly, in the recent report by Ma et al., no off-target effects were detected by WGS in any of the examined blastomeres of wild-type embryos [10]. An off-target effect was defined as one that differed from the on-target sequence by up to 7-nucleotide mismatches or up to 5-nucleotide mismatches with a 2-nucleotide DNA bulge. The authors attribute this high fidelity to injection of oocytes with recombinant Cas9 protein instead of plasmid transfection with Cas9 overexpression, so the enzymatic exposure time was likely much shorter than other studies that have reported high rates of offtarget DNA damage. This result should be interpreted with cautious optimism and data from larger studies are required to confirm that such fidelity can be reproduced with other monogenic conditions.

If this technology is implemented in clinical care, further assessment of off-target effects would need to be undertaken. Some questions include: (1) When should the assessment occur during embryonic development given the issue of mosaicism discussed above?; (2) What location and how many areas must be tested for clinical validation?; and (3) Which testing and bioinformatics platforms will provide adequate depth and resolution for quantifying indels and off-target effects and likewise exclude the possibility of allele dropout?

\section{Questions about the future}

In the era of personalized medicine in reproductive health, the appeal of genome editing is evident. However, concerns loom large - from the ethical ones regarding the potential for misapplication to the scientific ones regarding efficiency of correction, indels, and off-target effects.

The scientific community is already moving forward with CRISPR-Cas9. REIs and our professional societies have been left on the sideline, or are choosing to remain silent. We should be the ones leading this debate; after all, any clinical implementation of this technology will no doubt occur on our home field.

\section{References}

1. Anonymous. Conception in a watch glass. N Engl J Med 1937;217: 678. Retrieved from: http://www.nejm.org/doi/full/10.1056/ NEJM193710212171709.

2. Liang P, Xu Y, Zhang X, et al. CRISPR/Cas9-mediated gene editing in human tripronuclear zygotes. Protein Cell. 2015;6:363-72.

3. Kang X, He W, Huang Y, et al. Introducing precise genetic modifications into human 3PN embryos from CRISPR/Cas-mediated genome editing. J Assist Reprod Genet. 2016;33:581-8.

4. Tang L, Zeng Y, Du H, et al. CRISPR/Cas9-mediated gene editing in human zygotes using Cas 9 protein. Mol Gen Genomics. 2017;292:525-33.

5. National Academies of Sciences, Engineering, and Medicine. Human genome editing: science, ethics, and governance. Washington, DC: The National Academies Press; 2017. 
6. Ethics committee of the American Society of Reproductive Medicine. Informed consent and the use of gametes and embryos for research: a committee opinion. Fertil and Steril. 2014;101:332-5.

7. Miller JC, Tan S, Qiao G, et al. A TALE nuclease for efficient genome editing. Nat Biotechnol. 2011;29:143-8.

8. Mussolino C, Morbitzer R, Lutge F, et al. Nucleic Acids Res. 2011;39:9283-93.
9. Maeder ML, Thibodeau-Beganny S, Osiak A, et al. Mol Cell. 2008;31:294-301.

10. Ma H, Marti-Gutierrez N, Park SW, et al. Correction of a pathogenic gene mutation in human embryos. Nature. 2017; https://doi.org/ 10.1038/nature23305.

11. Egli D, Zuccaro MV, Kosicki M, Church GM, Bradley JM. Interhomologue repair in fertilized human eggs? bioRxiv. 2017; https:// doi.org/10.1101/181255. 